\title{
Laparoscopic Excision of a Paraganglioma Located in the Renal Arte- riovenous Window - A Reasonable Approach
}

\author{
Eugene Lin ${ }^{1,2,3}$, Ho Lin², Mao-Sheng Lin ${ }^{3}$, Jungle C.H. Wu ${ }^{3}$, Wen-Chi Chen ${ }^{4}$ and Allen W. Chiu ${ }^{*}{ }^{4,5}$
}

${ }^{1}$ Department of Urology, Chang Bing Show Chwan Hospital, ${ }^{2}$ Department of Life Science, National Chung Hsing University, ${ }^{3}$ Graduate Institute of Chinese Medical Science, China Medical University, ${ }^{4}$ Division of Urology, Department of Surgery, Taipei City Hospital, Zhongxiao Branch, ${ }^{5}$ National Yang-Ming University, School of Medicine, Taiwan

\begin{abstract}
Background: Paragangliomas are rare tumors that arise from extra-adrenal chromaffin cells. The occurrence of these tumors between the renal artery and the renal vein is very rare. Here we report the case of a 60-year-old woman with a solitary kidney who was incidentally found to have a paraganglioma without any endocrine dysfunction.

Methods: Under general anesthesia, the renal hilum was explored, and a tumor was identified between the renal artery and the renal vein. The tumor was removed completely with laparoscopy.

Results: Preoperative CT-guided biopsy revealed pheochromocytoma. We excised the tumor laparoscopically and pathological diagnosis was paraganglioma. Histological analysis revealed that the tumor was composed of chromaffin cells in a predominantly alveolar growth pattern.

Conclusion: This patient was followed up at our out-patient-department for two and a half years. The plasma creatinin and the kidney size were normal. There was no tumor recurrence under ultrasonography examination. Laparoscopic excision of paraganglioma reduces postoperative pain, facilitates early recovery and shortens the hospital stay.
\end{abstract}

Keywords: Paraganglioma, pheochromocytoma, adrenal tumor.

\section{INTRODUCTION}

Paragangliomas are rare tumors arising from extra-adrenal chromaffin cells or neuroectodermal chemoreceptor. They are highly vascularized and sometimes can be functional, secreting catecholamines and other peptides. They occur anywhere from the skull base to the pelvic floor, within the paraganglion system [1]. They are extra-adrenal pheochromocytomas that are often located in the retroperitoneum in the periaortic region $[2,3]$. The signs and symptoms of a pheochromocytoma are those of the sympathetic nervous system hyperactivity and can also cause resistant arterial hypertension.

Traditionally, open surgical procedures were used to remove paragangliomas. However, recent improvements in preoperative imaging and advanced laparoscopic instruments have made laparoscopic resection of paragangliomas feasible and safe. Recently, Bryan et al. reported laparoscopic resection of periaortic paragangliomas in four patients [4]. Here we report the case of a 60 -year-old woman with a solitary kidney who was accidentally found to have a paraganglioma without any endocrine dysfunction. The tumor was found to be between the renal artery and the renal vein which is very rare. We describe the presentation and imaging findings of this patient.

\section{MATERIALS AND METHODS}

A 60-year-old woman with a history of right ureteral transitional cell carcinoma (TCC) and with a post nephrouretectomy eight years ago was admitted to our hospital.

*Address correspondence to this author at the Division of Urology, Department of Surgery, Taipei City Hospital, Zhongxiao Branch, Taipei, Taiwan; Tel: 886-933090403; Fax: 886-4-7629188;

E-mail:s0924.tw@yahoo.com.tw
She had hypertension for more than ten years. The average systolic pressure after medication control was $150 \mathrm{mmHg}$. She was being followed-up regularly at our outpatient department every six months. One day, when she had a renal ultrasonography as routine follow up for TCC, a suprarenal tumor was noted. CT scan revealed that the tumor was located in the left suprarenal space. 64-slice CT was then performed which revealed the tumor mass just between the renal artery and the renal vein. Preoperative CT-guided biopsy was done. Alpha-adrenergic blockers were given to the patient for three days for preventing intra-operative hypertension crisis.

Laparoscopic tumor excision was performed for this extra-adrenal pheochromocytoma. Under general anesthesia, the patient was placed in supine position. Four trocars were used - a $10 \mathrm{~mm}$ umbilical trocar for the camera, a $10 \mathrm{~mm}$ trocar on the left flank, a 5-mm trocar at the midline of the abdomen and another 5-mm trocar at the left upper abdominal quadrant for instrument and retraction. Mobilization of the transverse colon, descending colon, pancreas tail and spleen were done. After the retroperitoneal space was exposed, the left kidney was mobilized. The renal hilum was explored, and a tumor was identified between the renal artery and the renal vein. Feeding vessels were controlled with clips and hemostasis was secured. The tumor was excised completely with Ultrasonic Harmonic Scalpel (Ethicon Endosurgery, Cincinnati, OH, USA) and Bipolar System (Gyrus-ACMI PK system). A part of the excised tumor was fixed in formalin and send to the pathologist for immunohistochemical analyses.

Fixed sections of the tumor was embedded in paraffin on edge to provide a cross sectional view of the specimen after 
microtome sectioning. Five- micron thick sections were mounted on positively charged slides, deparaffinized in xylene, stained with hematoxylin- eosin for light microscopy. Immunohistochemistry was performed using the following antibodies, chromogranin A (1:20; Biogenex, San Ramon, CA, USA) and S- 100 protein (1:1000, Dako, Carpinteria, CA, USA).

\section{RESULTS}

A $3.6 \times 3.7 \mathrm{~cm}$ retroperitoneal mass was found incidentally by renal ultrasonography (Fig. 1). CT scan showed an enhanced tumor $4.5 \times 3.5 \times 4.0 \mathrm{~cm}$ in size (Fig. 2). The tumor was located in the left suprarenal space indicating an adrenal tumor. 64-slice CT revealed that the tumor mass was just between the renal artery and the renal vein (Figs. 3A, B). Preoperative CT-guided biopsy revealed pheochromocytoma. Urine catecholamine was $3.44 \mathrm{mg} / 24 \mathrm{hr}$ (normal 1.0$7.5 \mathrm{mg} / 24 \mathrm{hr}$ ) and plasma renin was $3.03 \mathrm{~mol} / \mathrm{L}$ (normal 0.5 $5.7 \mathrm{~mol} / \mathrm{L})$.

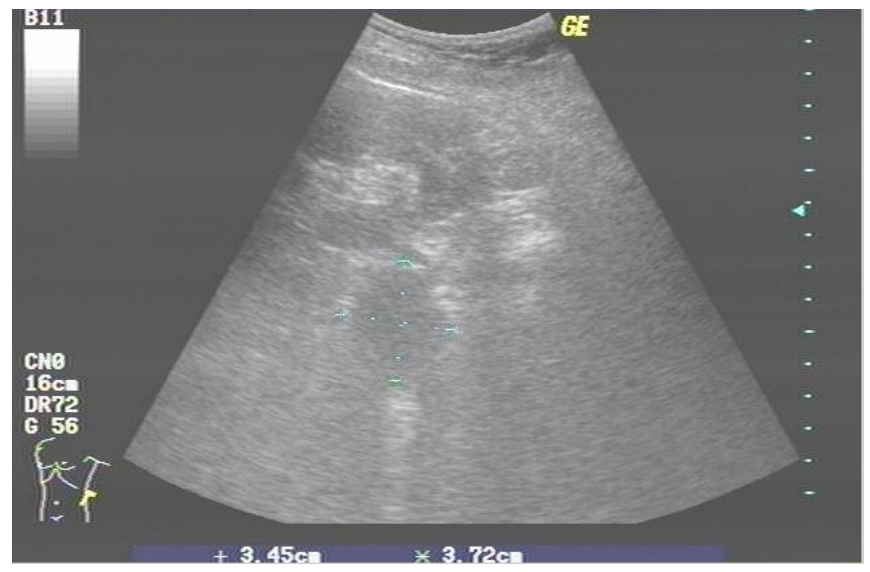

Fig. (1). A 3.6x3.7 cm retroperitoneal mass which was noted incidentally by renal ultrasonography.

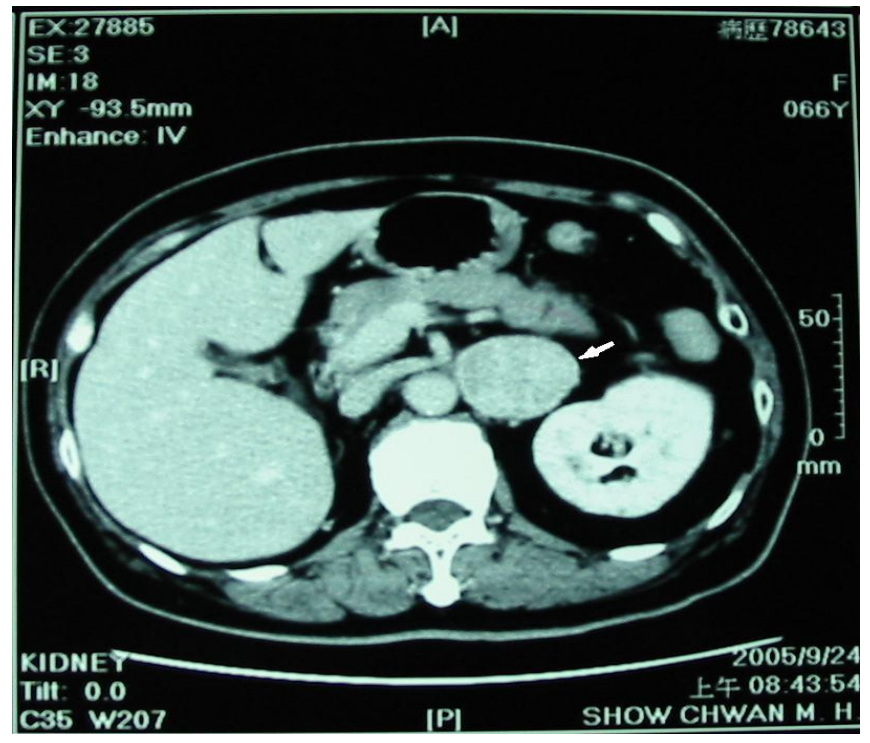

Fig. (2). Computed tomogram showing a large and enlarged tumor mass (arrow) about $4.5 \times 3.5 \times 4.0 \mathrm{~cm}$ in size. The tumor was located at left suprarenal space indicating an adrenal tumor.

The tumor was removed completely with laparoscopy. Fig. (4) shows the resected tumor. The total operation time was 300 mins. Estimated blood loss was $300 \mathrm{ml}$. The patient had an uneventful postoperative course and was discharged after 6 days.

(A)

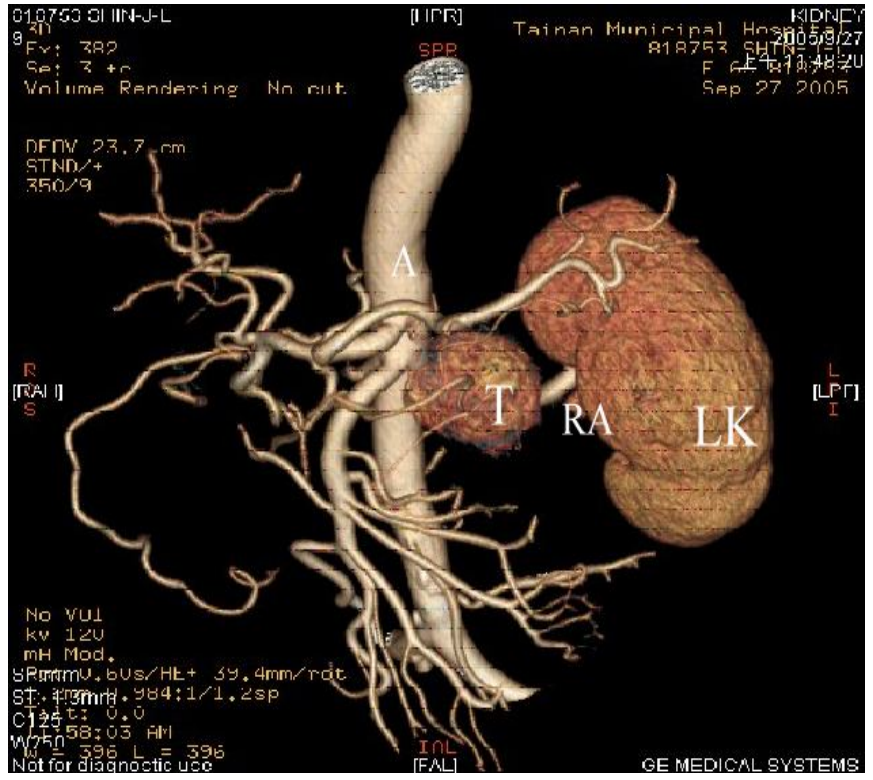

(B)

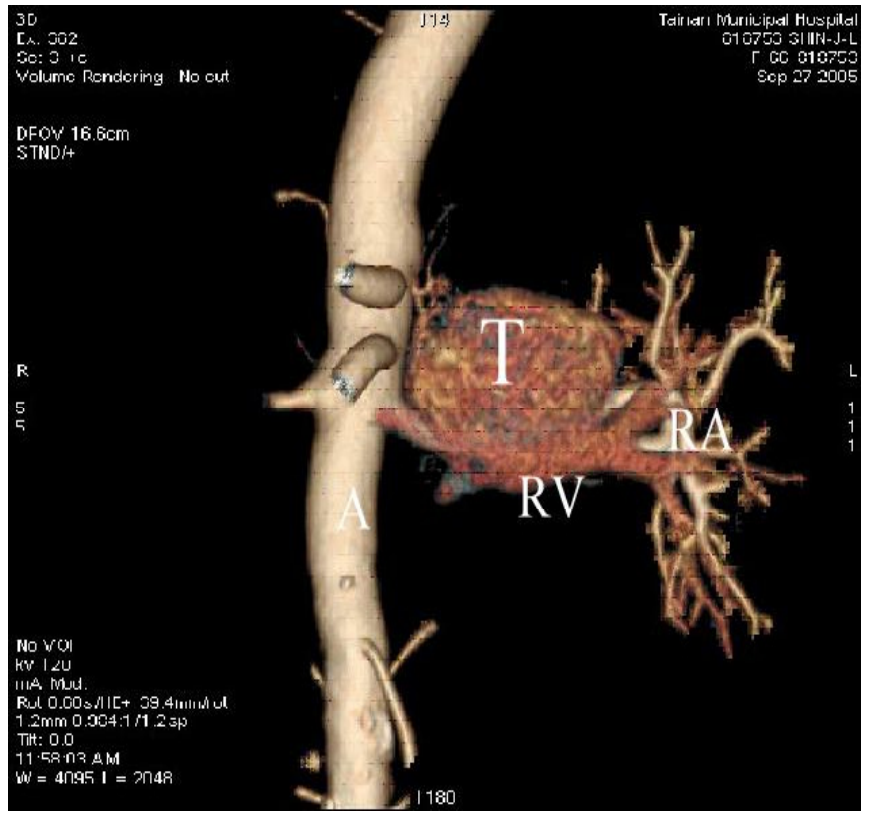

Fig. (3). (A) and (B) 64-slice CT with 3D reconstruction, showing the tumor mass $(\mathrm{T})$ seen between renal artery (RA) and renal vein (RV). (A represents aorta and LK represents left kidney).

Pathology confirmed the diagnosis of paraganglioma. The specimen consisted of an encapsulated tumor, associated with adrenal gland. The tumor with adrenal gland weighed $24 \mathrm{gm}$ and measured $3.5 \times 3 \times 3 \mathrm{~cm}$ (Fig. 4). The tumor was composed of chromaffin cells with granular and basophilic to amphophilic cytoplasm in a predominantly alveolar (zellballen) growth pattern. There was prominent cellular and nuclear pleomorphism (Fig. 5A, B). Immunohistochemistry revealed that the tumor cells were strongly positive to chromogranin A (Fig. 5C). S-100 protein was detected by the 
supportive cells that surround the nests (Fig. 5D). These findings are consistent with paraganglioma. The stroma was vascular-rich and with focal sclerotic changes. There were rare mitotic figures. No adrenal cortical tissue was observed in the tumor. The tumor was separated by adipose tissue from the adjacent adrenal gland with a well defined fibrous capsule.

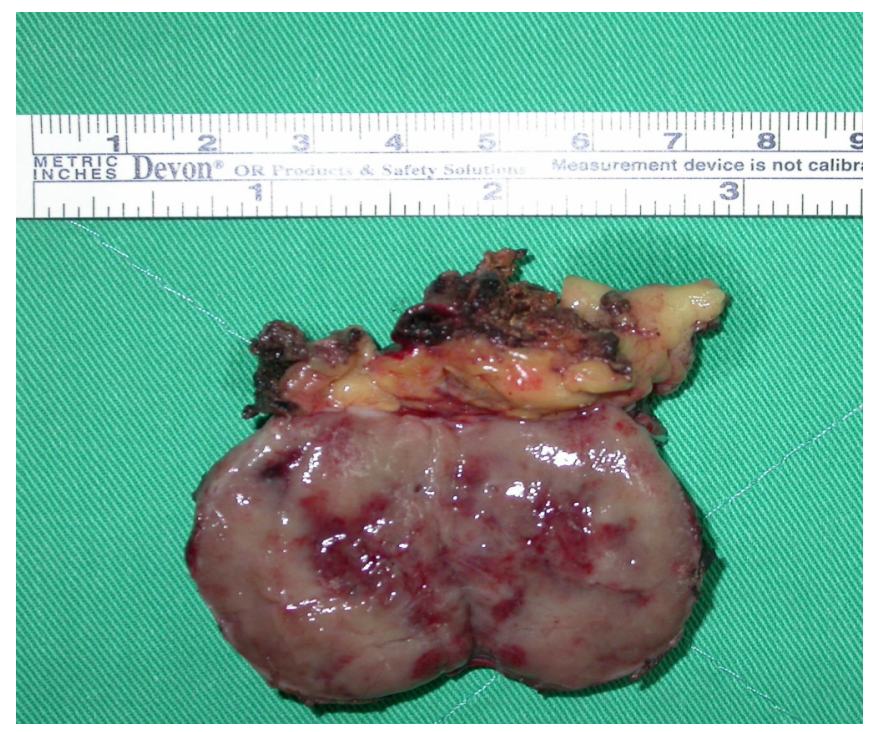

Fig. (4). Photograph of the resected specimen consisting of an encapsulated tumor $(\mathrm{T})$ sized in $3.5 \times 3 \times 3 \mathrm{~cm}$, associated with an adrenal gland (A).

This patient was followed up at our out-patientdepartment every 3 months for two and a half years. The

(A)

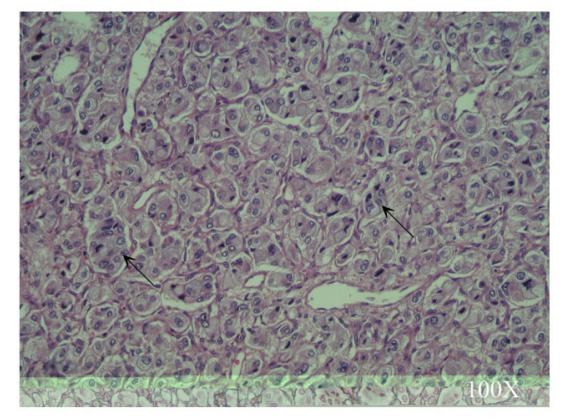

(C)

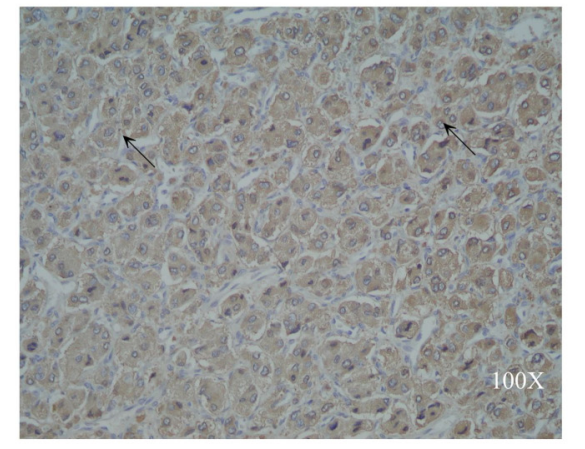

plasma creatinin was $1.3 \mathrm{mg} / \mathrm{dl}$ (normal range $0.5-1.5 \mathrm{mg} / \mathrm{dl}$ ) in postoperative first year and $1.2 \mathrm{mg} / \mathrm{dl}$ in the postoperative second and third year. The average systolic pressure after single medication control was $140 \mathrm{mmHg}$ in postoperative first year and $136 \mathrm{mmHg}$ in the postoperative second and third year. The renal ultrasonography revealed kidney size in $10.8 \times 6.5 \mathrm{~cm}$ at first year follow up, which remain the same size in the following two years. There was no tumor recurrence under ultrasonography examination.

\section{DISCUSSION}

Paragangliomas are extra-adrenal pheochromocytomas often located in the retroperitoneum along the sympathetic chain. Laparoscopic resection is recommended for adrenal tumor. Paragangliomas have higher malignancy potential than adrenal pheochromocytomas. Malignancy rates of 29 to 40 percent have been reported [5]. However, the histologic criteria for malignancy remain difficult. The number of mitosis, nuclear atypia, and either capsular or vascular invasion may not be able to predict malignancy. It can be determined by the presence of metastasis. Since metastasis is more common with paraganglioma, laparoscopic examination of the peritoneum before tumor excision is mandatory. Wide mobilization of the colon, mesentery and adjacent organs is necessary for adequate exposure. Laparoscopy has the advantage of optical magnification and provides better visualization of small vessels, which allows for meticulous dissection during tumor excision [6]. Tumors located between major vessels rarely invade these vessels, but careful and meticulous dissection of the surrounding small vessels is necessary. For the smaller tumor, laparoscopic ultrasonography may be helpful in identifying the anatomy dur-

\section{(B)}

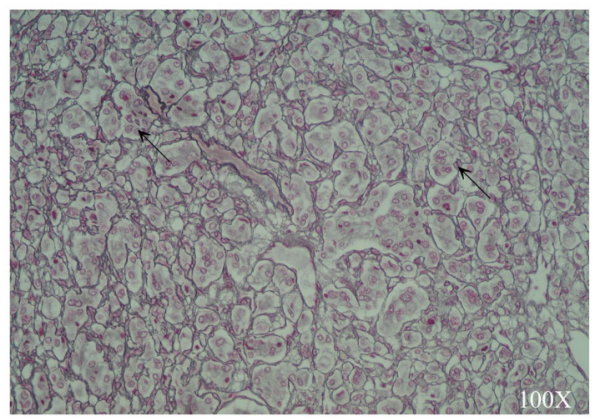

(D)

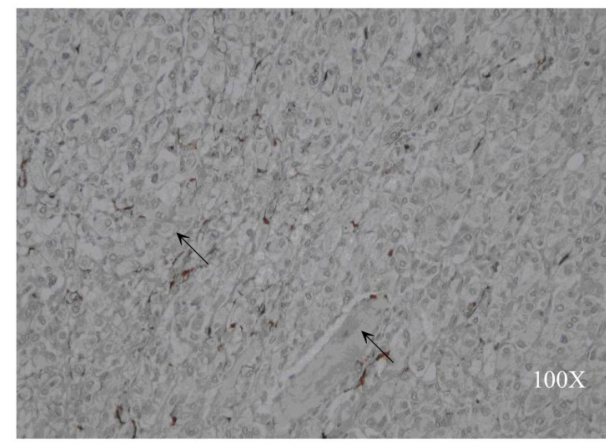

Fig. (5). (A) and (B) The paraganglioma showing the characteristic "zellballen" architecture in hematoxylin \& eosin staining (arrows). Note that the tumor cells were polyhedral with round or oval nuclei. (C) Intense immunoreactivity for chromogranin A in the tumor cells of paraganglioma (arrows). (D) Sustentacular cells immunostained for S-100 protein at the periphery of the tumor cell nests of paraganglioma (arrows). All figures are in 100X magnification. 
ing the operation [7]. Laparoscopic excision of paraganglioma reduces postoperative pain, facilitates early recovery and shortens the hospital stay as compared with open surgery [8].

With the introduction of 64-slice CT with a slice thickness of $2 \mathrm{~mm}$, it is now possible to employ three dimensional CT datasets for reconstruction in a free imaging plane without loss of quality. With the advent of faster scanning techniques it is now possible to separate arterial blood flow from venous flow; to scan the tumor just between the major vessels demonstrating the complex anatomy in multiple planes $[9,10]$. Both CT and magnetic resonance imaging (MRI) are highly sensitive for determining the tumor location, tumor number and presence of metastasis. With CT, there is some exposure to radiation and a slight risk of exacerbation of hypertension if a radiographic contrast agent is given. However, it can be prevented by pretreatment with alpha-adrenergic blockade [11]. With MRI, there is neither radiation nor dye, but it is more expensive. Even with the advent of the 64-slice CT, the MRI T2-weighted image can still provide useful information for differentiating pheochromocytoma and paraganglioma from other adrenal tumors [12]. On MRI T2-weighted images, pheochromocytomas and paragangliomas appear hyperintense, while other adrenal tumors are isointense when compared with the liver. When multiple tumors are detected on CT or MRI, an MIBG scan will be needed. MIBG is a compound resembling norepinephrine that is taken up by adrenergic tissue, and pentatreotide is taken up by tissues rich in somatostatin receptors, such as adrenergic tissue [13].

Paragangliomas are classified into functional and nonfunctional by the presence of clinical symptoms such as hypertension or hyperglycemia, which are characterized by the secretion of catecholamines [14]. The patient whom we reported did not show any accompanying clinical symptoms and the tumor was found incidentally during the regular follow-up ultrasonography for TCC. Most patients with nonfunctional tumors have pain and occasionally have symptoms of metastases. Functional tumors are easier to diagnose because catecholamines in urine are elevated, usually with an elevated level of norepinephrine [15]. The biochemical diagnosis of functional adrenal pheochromocytoma or extra-adrenal paraganglioma is based on excessive secretion of catecholamines and their metabolites [16]. Our patient had the catecholamine level within the normal range and hence the paraganglioma was not detected earlier.

The plasma creatinin and the average systolic pressure were within the normal range and the kidney size was normal in the postoperative years. These results demonstrate that the kidney vessels or function was not damaged during the laparoscopic operation. There was no tumor recurrence under ultrasonography examination.

Improvements in preoperative imaging and laparoscopic instruments have made laparoscopic resection of paragangliomas feasible and safe. Our patient was with a solitary kidney and the paraganglioma was detected during a routine follow up.
The tumor was between the renal artery and vein which is never reported before according to our knowledge. Our patient having a solitary kidney only and the tumor between the major blood vessels, laparoscopic excision of the tumor was high risk than in a normal person. Even then we were able to excise the tumor successfully and the patient is remaining healthy for the past three years. The laparoscopic method we used to excise the tumor has many advantageous over the traditional methods, such as minimal blood loss, decreased pain, and shorter hospital stay. This method has comparable results with the traditional open surgery.

\section{ACKNOWLEDGEMENTS}

The authors acknowledge Shang-Tao Lin M.D. and Chien-Long Kuo M.D. for the histological analysis.

\section{REFERENCES}

[1] Whitson BA, Tuttle TM. Laparoscopic resection of periaortic paragangliomas. Am Surg 2005; 71: 450- 4.

[2] Erickson D, Kudva YC, Ebersold MJ, et al. Benign paragangliomas: clinical presentation and treatment outcomes in 236 patients. J Clin Endocrinol Metab 2001; 86: 5210-6.

[3] Whalen RK, Althausen AF, Daniels GH. Extra-adrenal pheochromocytoma. J Urol 1992; 147: 1-10.

[4] Bryan AW, Todd MT. Laparoscopic resection of periaortic paragangliomas. Am Surg 2005; 71: 450-5.

[5] Melicow MM. One hundred cases of pheochromocytoma (107 tumors) at the Columbia-Presbyterian Medical Center, 1926-1976: a clinicopathological analysis. Cancer 1997; 40: 1987-2004.

[6] Janetschek G, Finkenstent G, Gasser R, et al. Laparoscopic surgery for pheochromocytoma, partial resection, excision of paragangliomas. J Urol 1998; 160: 330-4.

[7] Kercher KW, Heniford BT, Marroum MC, Greene FL. Laparoscopic intraoperative ultrasonic localization and resection of an extra-adrenal pheochromocytoma. J Laparoendosc Adv Surg Tech 1999; 9: 511-5.

[8] Imai T, Kikumori T, Ohiwa M, Mase T, Funahashi H. A casecontrolled study of laparoscopic compared with open lateral adrenalectomy. Am J Surg 1999; 18: 50-3.

[9] Halpin SF. Brain imaging using multislice CT: a personal perspective. Br J Radiol 2004; 77: S20-26.

[10] Ehara M, Surmely JF, Kawai M, et al. Diagnostic accuracy of 64slice computed tomography for detecting angiographically significant coronary artery stenosis in an unselected consecutive patient population: comparison with conventional invasive angiography. Circ J 2006; 70: 564-71.

[11] Nobels FR, Kwekkeboom DJ, Coopmans W, et al. Chromogranin A as serum marker for neuroendocrine neoplasia: Comparison with neuron-specific enolase and the alpha-subunit of glycoprotein hormones. J Clin Endocrinol Metab 1997; 82: 2622-7.

[12] Bravo EL. Evolving concepts in the pathophysiology, diagnosis, and treatment of pheochromocytoma. Endocr Rev 1994; 15: 356-68.

[13] Bravo EL. Pheochromocytoma: New concepts and future trends. Kidney Int 1991; 40: 544-6.

[14] Yamaguchi T, Tada M, Takahashi H, et al. An incidentally discovered small and asymptomatic para-aortic paraganglioma. Eur Surg Res 2008; 40: 14-8

[15] Sclafani LM, Woodruff JM, Brennan MF. Extra-adrenal retroperitoneal paragangliomas: nature history and response to treatment. Surgery 1990; 108: 1124-30.

[16] Somasundar P, Krouse R, Hostetter R, Vaughan R, Covey T. Paragangliomas - A decade of clinical experience. J Surg Oncol 2000; 74: 286-90. 